\title{
In Vivo 7T MR Quantitative Susceptibility Mapping Reveals Opposite Susceptibility Contrast between Cortical and White Matter Lesions in Multiple Sclerosis
}

\author{
(D)W. Bian, (D) E. Tranvinh, (D). Tourdias, (D) M. Han, (D). Liu, (D). Wang, (D) B. Rutt, and (D).M. Zeineh
}

\begin{abstract}
BACKGROUND AND PURPOSE: Magnetic susceptibility measured with quantitative susceptibility mapping has been proposed as a biomarker for demyelination and inflammation in patients with MS, but investigations have mostly been on white matter lesions. A detailed characterization of cortical lesions has not been performed. The purpose of this study was to evaluate magnetic susceptibility in both cortical and WM lesions in MS by using quantitative susceptibility mapping.
\end{abstract}

MATERIALS AND METHODS: Fourteen patients with MS were scanned on a 7T MR imaging scanner with T1-, T2-, and T2*-weighted sequences. The $\mathrm{T} 2^{*}$-weighted sequence was used to perform quantitative susceptibility mapping and generate tissue susceptibility maps. The susceptibility contrast of a lesion was quantified as the relative susceptibility between the lesion and its adjacent normal-appearing parenchyma. The susceptibility difference between cortical and WM lesions was assessed by using a $t$ test.

RESULTS: The mean relative susceptibility was significantly negative for cortical lesions $\left(P<10^{-7}\right)$ but positive for WM lesions $\left(P<10^{-22}\right)$. A similar pattern was also observed in the cortical $(P=.054)$ and WM portions $(P=.043)$ of mixed lesions.

CONCLUSIONS: The negative susceptibility in cortical lesions suggests that iron loss dominates the susceptibility contrast in cortical lesions. The opposite susceptibility contrast between cortical and WM lesions may reflect both their structural (degree of myelination) and pathologic (degree of inflammation) differences, in which the latter may lead to a faster release of iron in cortical lesions.

ABBREVIATIONS: MPFLAIR = magnetization-prepared fluid-attenuated inversion recovery; QSM = quantitative susceptibility mapping

M ultiple sclerosis is a debilitating chronic inflammatory disorder of the central nervous system. MS pathogenesis is not fully understood and is thought to involve the whole brain. While previous MS imaging studies have been largely focused on white matter, recent investigations have focused on the importance of cortical gray matter damage, ${ }^{1}$ because cor-

Received December 21, 2015; accepted after revision April 4, 2016.

From the Departments of Radiology (W.B., E.T., B.R., M.M.Z.) and Neurology (M.H.), Stanford University School of Medicine, Palo Alto, California; Service de Neurolmagerie Diagnostique et Thérapeutique (T.T.), Centre Hospitalier Universitaire de Bordeaux, Bordeaux Cedex, France; Institut National de la Santé et de la Recherche Médicale U 862 (T.T.), Université de Bordeaux, Bordeaux Cedex, France; and Department of Radiology (T.L., Y.W.), Weill Medical College of Cornell University, New York, New York.

This work was supported by GE Healthcare (B.R. and M.M.Z.); National Institutes of Health grants P41EB015891 (B.R. and M.M.Z), S10RR026351 (B.R. and M.M.Z), and RO1NS090464 (Y.W.); and grants from Association pour l'aide à la Recherche contre la Sclérose en Plaques and Translational Advanced Imaging Laboratory (T.T). Please address correspondence to M.M. Zeineh, MD, PhD, Department of Radiology, Stanford University School of Medicine, Lucas Center for Imaging, Room P271, 1201 Welch Rd, Stanford, CA 94305-5488; e-mail: mzeineh@stanford.edu

- Indicates open access to non-subscribers at www.ajnr.org

http://dx.doi.org/10.3174/ajnr.A4830 tical lesions may be more relevant to physical and cognitive disability in patients than WM lesions. ${ }^{2}$ This difference raises questions about the various underlying pathologic aspects of cortical and WM lesions. Histochemical staining has shown that cortical lesions have a lower degree of inflammation ${ }^{3}$ and blood-brain barrier damage than WM lesions, ${ }^{4}$ implying that the cortical lesion may be partly independent of inflammation. ${ }^{5}$ Because it is useful to evaluate cortical and WM lesions in vivo across the whole brain, MR imaging is an important tool that complements histochemical staining.

While traditional water content- and proton mobility-based MR imaging modalities show similar abnormalities for both cortical and WM lesions, advanced high-field-strength MR imaging with susceptibility-weighted contrasts such as $\mathrm{R} 2 * / \mathrm{T} 2 *$ mapping and quantitative susceptibility mapping (QSM) may have the potential to discriminate features of cortical and WM lesions. In active and chronic WM lesions, 7T MR imaging studies have shown that regions with decreased $\mathrm{R} 2^{*}$ and increased magnetic susceptibility correspond to histologically verified regions with demyelination- and/or inflammation-associated iron accumulation. ${ }^{6-9}$ More recently, $\mathrm{R} 2^{\star} / \mathrm{T} 2^{\star}$ mapping at $7 \mathrm{~T}$ has shown de- 
Table 1: Patient demographic/clinical data and lesion counts ${ }^{\mathrm{a}}$

\begin{tabular}{|c|c|c|c|c|c|c|c|c|}
\hline & Sex & Age (yr) & $\begin{array}{c}\text { Disease } \\
\text { Duration (yr) }\end{array}$ & Treatment Type & $\begin{array}{c}\text { Minimum WM } \\
\text { Lesion Age (mo) }\end{array}$ & $\begin{array}{l}\text { Cortical } \\
\text { Lesion }\end{array}$ & WM Lesion & $\begin{array}{l}\text { Mixed } \\
\text { Lesion }\end{array}$ \\
\hline \multicolumn{9}{|l|}{ Patient } \\
\hline 1 & $\mathrm{~F}$ & 37 & 11 & Copaxone $^{\mathrm{b}}$ & 12 & 2 & 6 & 1 \\
\hline 2 & M & 42 & 12 & Copaxone & 10 & 1 & 11 & 0 \\
\hline 3 & $\mathrm{~F}$ & 42 & 3 & Copaxone & 8 & 0 & 2 & 0 \\
\hline 4 & $\mathrm{~F}$ & 30 & 3 & Tysabric $^{\mathrm{C}}$ & 9 & 5 & 23 & 1 \\
\hline 5 & $F$ & 49 & 2 & Rebif $^{d}$ & 18 & 7 & 13 & 1 \\
\hline 6 & $M$ & 32 & 6 & Copaxone & 10 & 3 & 30 & 4 \\
\hline 7 & $M$ & 42 & 1 & Copaxone & 7 & 1 & 5 & 0 \\
\hline 8 & $\mathrm{~F}$ & 33 & 1 & Copaxone & 10 & 0 & 4 & 0 \\
\hline 9 & $\mathrm{~F}$ & 44 & 15 & No Treatment & 6 & 0 & 4 & 1 \\
\hline 10 & $\mathrm{~F}$ & 31 & 1 & No Treatment & 3 & 0 & 12 & 0 \\
\hline 11 & $\mathrm{~F}$ & 41 & 16 & Tysabri & 4 & 2 & 15 & 1 \\
\hline 12 & $F$ & 58 & 25 & Copaxone & 6 & 6 & 7 & 0 \\
\hline 13 & M & 37 & 8 & Copaxone & 13 & 0 & 15 & 0 \\
\hline 14 & $M$ & 48 & 6 & Copaxone & 5 & 0 & 0 & 0 \\
\hline Mean & & $40.4 \pm 7.9$ & $7.9 \pm 7.2$ & & $8.6 \pm 4.0$ & $1.9 \pm 2.3$ & $10.5 \pm 8.4$ & $0.64 \pm 1.1$ \\
\hline Total & & & & & & 27 & 147 & 9 \\
\hline
\end{tabular}

a Patients 9 and 10 were not on any disease-modifying treatment at the time of their 7T scans. Patient 9 was on Tysabri, but it was stopped 6 months prior to her 7T scan. All patients had relapsing-remitting MS except patient 11, who was in a transitional stage between relapsing-remitting MS and secondary-progressive MS but was still being treated for relapsing-remitting MS. Patient 14 had lesions that all regressed before the 7T scan.

b Glatiramer acetate injection.

cNatalizumab.

${ }^{\mathrm{d}}$ Interferon $\beta$-la.

Table 2: Parameters for MR imaging sequences ${ }^{a}$

\begin{tabular}{lcccc}
\hline \multicolumn{1}{c}{ Parameters } & T2* SPGR & T1 WM-Nulled MPRAGE & T1 CSF-Nulled MPRAGE & T2 MPFLAIR \\
\hline Acquisition & $2 \mathrm{D}$ axial & 3D coronal & 3D coronal & 3D coronal \\
TR & $1200 \mathrm{~ms}$ & $8.3 \mathrm{~ms}$ & $3.9 \mathrm{~ms}$ & $8000 \mathrm{~ms}$ \\
TE & $17.7 \mathrm{~ms}$ & $3.7 \mathrm{~ms}$ & $8.5 \mathrm{~ms}$ & $109.8 \mathrm{~ms}$ \\
TI & $\mathrm{NA}$ & $680 \mathrm{~ms}$ & $1200 \mathrm{~ms}$ & $2135 \mathrm{~ms}$ \\
Flip angle & $60^{\circ}$ & $4^{\circ}$ & $6^{\circ}$ & $90^{\circ}$ \\
Bandwidth & $19.2 \mathrm{kHz}$ & $15.6 \mathrm{kHz}$ & $19.2 \mathrm{kHz}$ & $62.5 \mathrm{kHz}$ \\
FOV & 180 & 180 & 180 & 180 \\
Matrix & $384 \times 384$ & $180 \times 180$ & $224 \times 224$ & $224 \times 224$ \\
No. of sections & 90 & 256 & 256 & 256 \\
Resolution & $0.47 \times 0.47 \times 1 \mathrm{~mm}^{3}$ & $1 \times 1 \times 1 \mathrm{~mm}^{3}$ & $0.8 \times 0.8 \times 0.8 \mathrm{~mm}^{3}$ & $0.8 \times 0.8 \times 0.8 \mathrm{~mm}^{3}$ \\
Acceleration factor & ASSET 2 & ARC $1.5 \times 1.5$ & ASSET 2.5 & ARC $2 \times 2$ \\
Acquisition time (min:s) & $6: 39$ & $5: 54$ & $6: 20$ & $5: 48$ \\
\hline
\end{tabular}

Note:-ASSET indicates array spatial sensitivity encoding technique; SPGR, spoiled gradient-recalled; ARC, Autocalibrating Reconstruction for Cartesian; NA, not applicable.

${ }^{a}$ Two patients had a slightly different resolution for T2* SPGR. One (patient 4 in Table 1) had a resolution of $0.47 \times 0.47 \times 1.2 \mathrm{~mm}^{3}$ and the other (patient 11 in Table 1) had a resolution of $0.47 \times 0.47 \times 1.1 \mathrm{~mm}^{3}$.

creased $\mathrm{R} 2 * /$ increased $\mathrm{T} 2 *$ in cortical lesions, indicating loss of both iron and myelin. ${ }^{10-13}$

However, simultaneous assessment of the susceptibility contrast in both cortical and WM lesions has not been performed, in particular by using in vivo QSM, which can measure tissue magnetic susceptibility with high reproducibility, even in the cortex. ${ }^{14}$ Compared with other susceptibility-based imaging techniques such as phase imaging, susceptibilityweighted imaging, and $\mathrm{R} 2^{*} / \mathrm{T} 2^{*}$ mapping, the deconvolution inherent in QSM removes the interfering effects of the susceptibility sources external to a voxel and makes the susceptibility sources within the voxel quantifiable. ${ }^{15,16}$ In addition, the high SNR and resolution at a high field strength of $7 \mathrm{~T}$ increase the in vivo quantification quality of QSM to benefit the characterization of small cortical lesions.

The purpose of this study was to use QSM at 7T to measure and compare in vivo susceptibility contrast in both cortical and WM lesions and identify differences that may reflect the known pathologic difference between the 2 subtypes of lesions.

\section{MATERIALS AND METHODS Patients}

We recruited 15 patients with MS from February 2013 to August 2013 at the Stanford University MS clinic. Informed consent was obtained from each patient, and the study was approved by our institutional review board. An MS neurologist (M.H., with 10 years' experience) evaluated patients on the basis of their clinical presentations, investigative work-ups, and the McDonald criteria. ${ }^{17}$ While quantitative clinical metrics of disability were not available for our subjects, patient medications taken at the time of the study are shown in Table 1. One patient was excluded from analysis because of a dataacquisition error prohibiting QSM reconstruction.

\section{MR Imaging}

All MR images were performed on a 7T scanner (Discovery MR950; GE Healthcare, Milwaukee, Wisconsin) with a 32-channel phased array receive coil (Nova Medical, Wilmington, Massachusetts). The imaging protocol (Table 2) covering the supraten- 


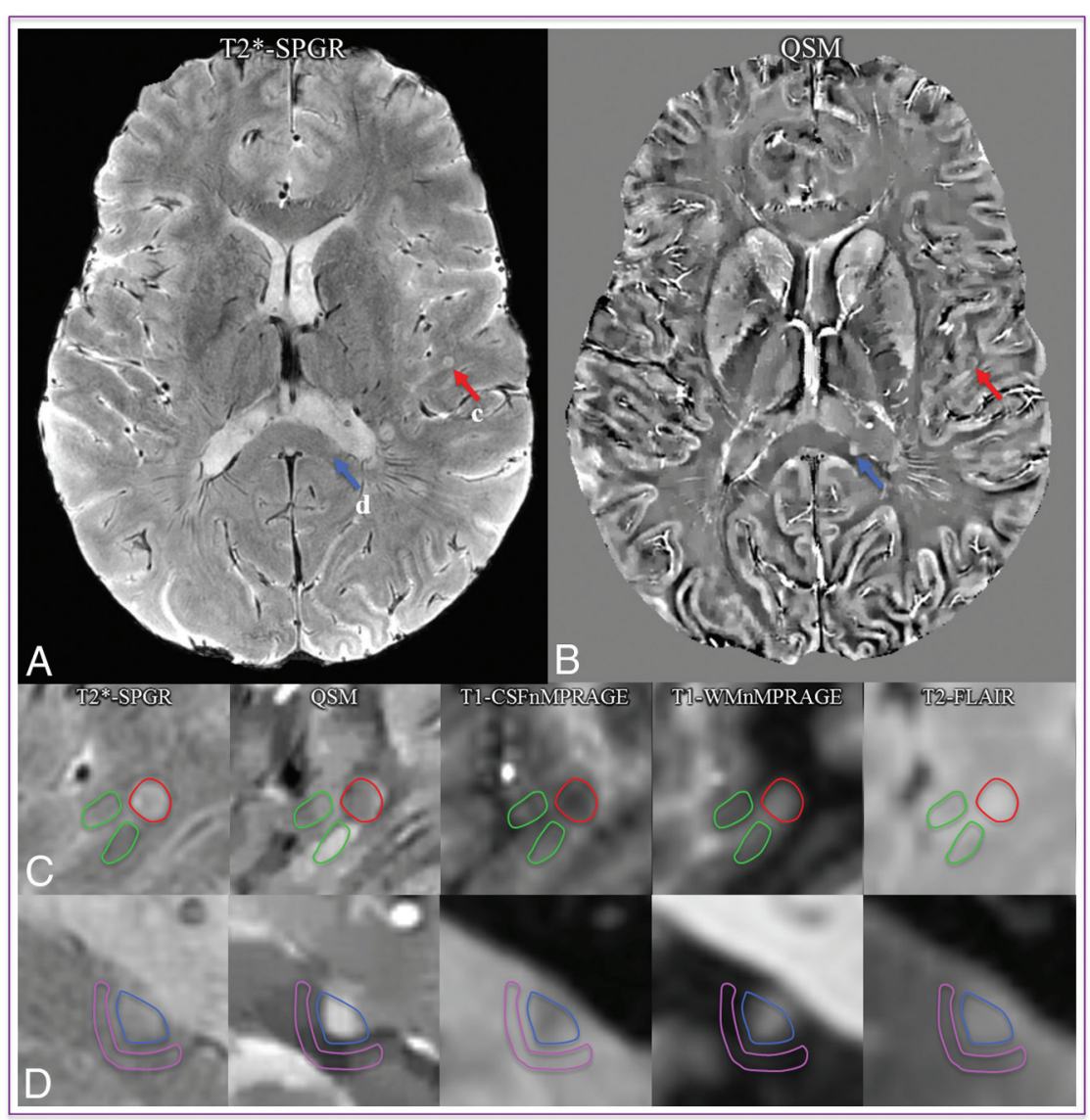

FIG 1. ROI definition. A whole section of magnitude $(A)$ and QSM images (B) show 1 cortical lesion (red arrows) and 1 WM lesion (blue arrows). C, The ROls of the cortical lesion and its adjacent normal-appearing cortical gray matter counterpart are delineated in red and green lines, respectively. $D$, The ROls of the WM lesion and its adjacent normal-appearing white matter counterpart are delineated in blue and pink lines, respectively. ROls were first defined on T2*-spoiled gradient-recalled images and then transferred to the other coregistered images. The gap between the lesion ROIs and the adjacent normal-appearing parenchyma reduces the partial volume effect in the segmentation. CSFnMPRAGE indicates CSF-nulled MPRAGE; WMnMPRAGE, WM-nulled MPRAGE.

torial brain included the following: 1) a $\mathrm{T} 2{ }^{*}$-weighted multisection $2 \mathrm{D}$ fast spoiled gradient-recalled sequence, 2 ) a coronal T1-weighted 3D WM-nulled MPRAGE sequence, ${ }^{18} 3$ ) a coronal T1-weighted 3D CSF-nulled MPRAGE sequence, and 4) a coronal 3D T2-weighted magnetization-prepared fluid-attenuated inversion recovery (MPFLAIR) sequence. ${ }^{19}$ The T1- and T2weighted images were acquired to aid in lesion identification and segmentation. The $\mathrm{T} 2{ }^{*}$-spoiled gradient-recalled images were first reconstructed into both magnitude and phase images, and then QSM images were computed by using the morphology-enabled dipole inversion method, ${ }^{20}$ which performs Laplacian phase unwrapping first, followed by phase deconvolution by using $\mathrm{L}_{1}$-norm minimization. To reduce the artifacts at the edge of brain while preserving as much as possible of the cortex, we eroded the unwrapped phase by $2.5 \mathrm{~mm}$ ( $\sim 5$ pixels) before the deconvolution. For each patient, the T1-WM-nulled MPRAGE, T1-CSF-nulled MPRAGE, and T2-MPFLAIR images were rigidly coregistered to the $\mathrm{T} 2^{\star}$-magnitude images by using the FMRIB Linear Image Registration Tool (FLIRT; http://www.fmrib.ox. ac.uk/) in FSL ${ }^{21}$ with a mutual information cost function.

\section{Lesion Identification and Segmentation}

All images were examined by raters and determined to be of adequate quality for MS lesion detection and characterization. MS lesions were defined as having abnormal signal on all traditional imaging sequences (hypointense on T1-CSFnulled MPRAGE and hyperintense on T2* ${ }^{*}$ spoiled gradient-recalled, T1-WMnulled MPRAGE, and T2-MPFLAIR) by the most votes from 3 experienced MS imaging investigators who reviewed images independently and were blinded to QSM images (E.T., neuroradiologist with 6 years' experience; W.B., neuroimaging scientist with 6 years' experience; M.M.Z., neuroradiologist, with 11 years' experience). Only lesions of $\geq 2$ $\mathrm{mm}$ were identified. All available $3 \mathrm{~T}$ clinical scans before the current $7 \mathrm{~T}$ scan were evaluated to identify whether any WM lesions were new or enhancing. The gray-white matter boundary on the T1CSF-nulled MPRAGE images was used to distinguish WM, cortical, or mixed cortical-WM lesions: All WM lesions were completely within the WM, all cortical lesions were primarily $(>75 \%)$ within the cortex, and all mixed lesions were $25 \%-75 \%$ within both the WM and cortex.

On $\mathrm{T}^{\star}$-spoiled gradient-recalled images, ROIs covering all hyperintense voxels were manually drawn jointly by W.B. and E.T. on multiple continuous image sections. WM and cortical lesion ROIs were drawn only within the WM and cortex, respectively, and each mixed lesion had 2 adjacent ROIs defined separately in its cortical and WM portions. Reference ROIs were drawn on adjacent normal-appearing WM for WM lesions or adjacent normal-appearing GM for cortical lesions. These normal-appearing ROIs were delineated from a single central section that contained the lesion (Fig 1). A donut-shaped region of adjacent homogeneous WM was used for normal-appearing WM; a homogeneous region of adjacent cortex continuous with both sides of the lesion was used for the normal-appearing GM. For a mixed lesion, 2 adjacent normal-appearing ROIs were defined separately for their corresponding normal-appearing cortical and WM portions. The adjacent normal-appearing ROI was within a 10-pixel vicinity of the lesion. A small gap was left between the lesion ROI and its adjacent normal-appearing ROI to reduce potential partial volume artifacts. After all ROIs had been segmented, the ROIs of lesions were overlaid on QSM images for a final quality control evaluation. Any blood vessels in the ROIs were removed, and any cortical or mixed lesions that were eroded or contaminated with artifacts due to QSM postprocessing were excluded from analysis. 
The susceptibility contrast of a lesion was quantified as the relative susceptibility between the lesion and its normal-appearing parenchyma, which was calculated by subtracting the mean susceptibility in the normal-appearing ROI from that in the lesion ROI.

\section{Statistics}

The relative susceptibility values for the set of cortical lesions, cortical portions of mixed lesions, WM lesions, and the WM portions of mixed lesions were each compared with zero by using the 1 -sample $t$ test. The relative susceptibility values in all cortical and WM lesions in the same subject were also averaged respectively; then, the above $t$ test were repeated. The statistical significance threshold was set as $P<.05$, with multiple comparisons corrected by the Bonferroni method.

\section{RESULTS}

Of the 14 patients $(40.4 \pm 7.9$ years of age, $7.9 \pm 7.2$ years of disease duration; see more detail in Table 1), 13 patients had relapsing-remitting MS, while 1 patient had relapsing-remitting MS but was transitioning to secondary-progressive MS. Twelve patients were undergoing disease-modifying therapy. A total of 183 lesions were identified (after removing 1 cortical lesion that contained notable artifacts on the QSM image): 27 (14.8\%) cortical, 147 (80.3\%) WM, and 9 (4.9\%) mixed. Eight of the 14 patients had cortical lesions (Table 1). Prior clinical 3T MR images indicated that all WM lesions were older than 3 months, and 8 of them (all from patient 4 ) were once contrastenhancing $>9$ months before the $7 \mathrm{~T}$ scan (Table 1), suggesting (but not proving) that none of the WM lesions in our sample were acute. Cortical lesions could not be reliably identified on prior 3T clinical images, and their ages were not determined. All patients were clinically stable between the time of the prior scan and the 7T scan.

The mean relative susceptibility values for $147 \mathrm{WM}$ and 27 cortical lesions were $0.014 \pm 0.014 \mathrm{ppm}$ and $-0.018 \pm 0.013$ ppm, respectively. The relative susceptibility value was positive for 132 of the 147 (89.8\%) WM lesions, but negative for 25 of the $27(92.6 \%)$ cortical lesions (Figs $2 \mathrm{~A}$ and 3 ). Of 2 cortical lesions whose susceptibility was positive, one had a susceptibility of 0.008 ppm (with a dark center and an asymmetric bright rim, Fig 4) and the other had a susceptibility that was almost zero (0.0004 ppm). The mean relative susceptibility value was significantly higher than zero for WM lesions $\left(P<10^{-22}\right)$ but significantly lower than zero for cortical lesions $\left(P<10^{-7}\right)$ (Table 3$)$.

After we averaged the susceptibility across lesions within each patient, all 13 patients had positive average relative susceptibility values for WM lesions $(0.014 \pm 0.010 \mathrm{ppm})$, and 7 of the 8 patients with cortical lesions had a negative average relative susceptibility value for cortical lesions $(-0.015 \pm$ $0.009 \mathrm{ppm}$ ) (Fig 2B). The patient with a positive average relative cortical susceptibility value for cortical lesions had only 1 cortical lesion (Fig 4). This patient-averaged relative susceptibility value was again significantly higher than zero $(P<.0004)$ for WM lesions but significantly lower than zero $(P<.004)$ for cortical lesions (Table 3).

The relative susceptibility values for WM and cortical portions in 9 mixed lesions were $0.014 \pm 0.018 \mathrm{ppm}$ and $-0.009 \pm 0.012$
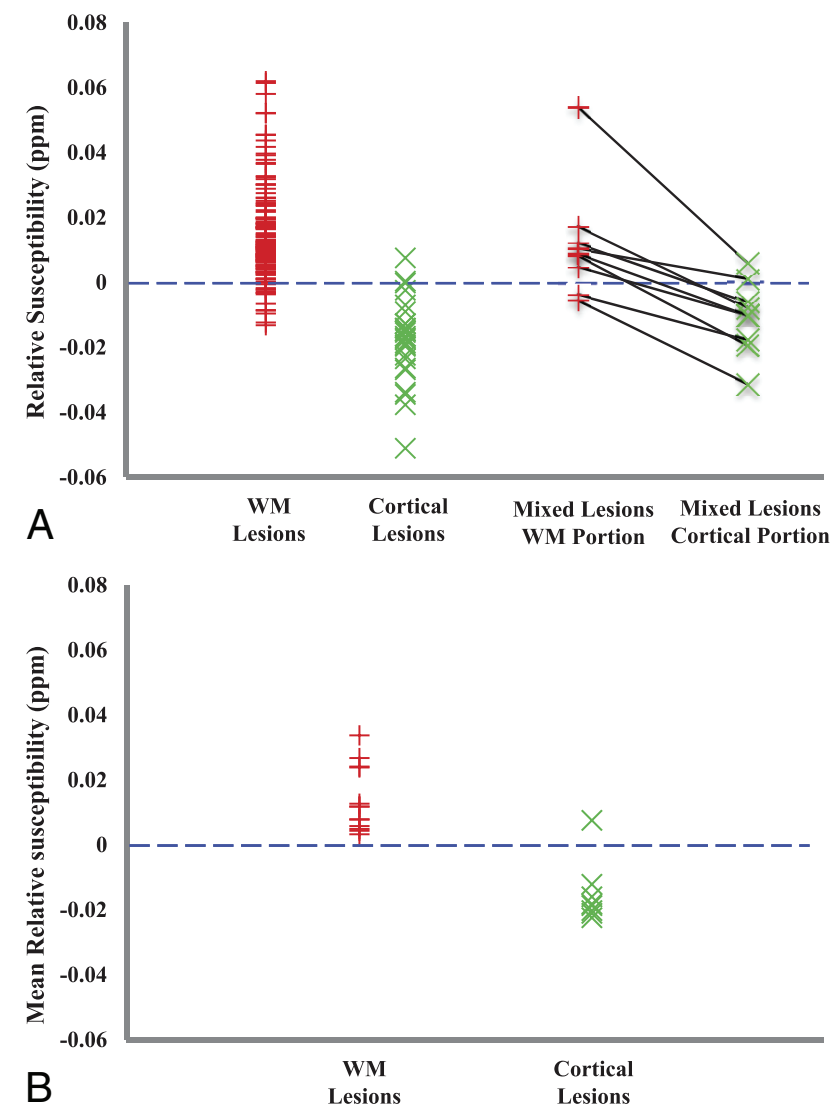

FIG 2. Relative susceptibility in MS lesions. $A$, The relative susceptibility in each individual lesion. Each black line on the right connects a pair of WM and cortical portions in a mixed lesion. $B$, The mean relative susceptibility after averaging the relative susceptibility across all lesions per type for each patient (13 patients had WM lesions, and 8 patients had cortical lesions).

ppm, respectively. All 9 mixed lesions had higher relative susceptibility values in their WM portions compared with their cortical counterparts. Seven of the $9(77.8 \%)$ lesions had positive relative susceptibility values in their WM portions $(P=.043)$, and the same percentage of lesions had negative relative susceptibility values in their cortical portions $(P=.054)$ (Figs $2 A$ and 5 and Table 3 ).

\section{DISCUSSION}

Our data demonstrate that the magnetic susceptibility values relative to normal-appearing adjacent parenchyma are negative for cortical lesions but positive for WM lesions, and a similar pattern was also found in the cortical and WM portions of mixed lesions, consistent with a recent postmortem study. ${ }^{22}$ The divergent contrast between cortical and WM lesions on QSM images cannot be revealed by using traditional MR imaging contrasts, including T2, $\mathrm{T} 1$, and $\mathrm{T} 2 *$.

\section{Positive Relative Susceptibility of WM Lesions}

Our observation of positive relative susceptibility for WM lesions is in line with data from previous studies, in which most WM lesions appeared QSM hyperintense/isointense relative to normal-appearing WM. ${ }^{9,23}$ Demyelination (loss of diamagnetic myelin) has been identified as a contributor to the increased susceptibility. ${ }^{6-8}$ Accumulation of highly paramagnetic iron is also often found in microglia/macrophages near 

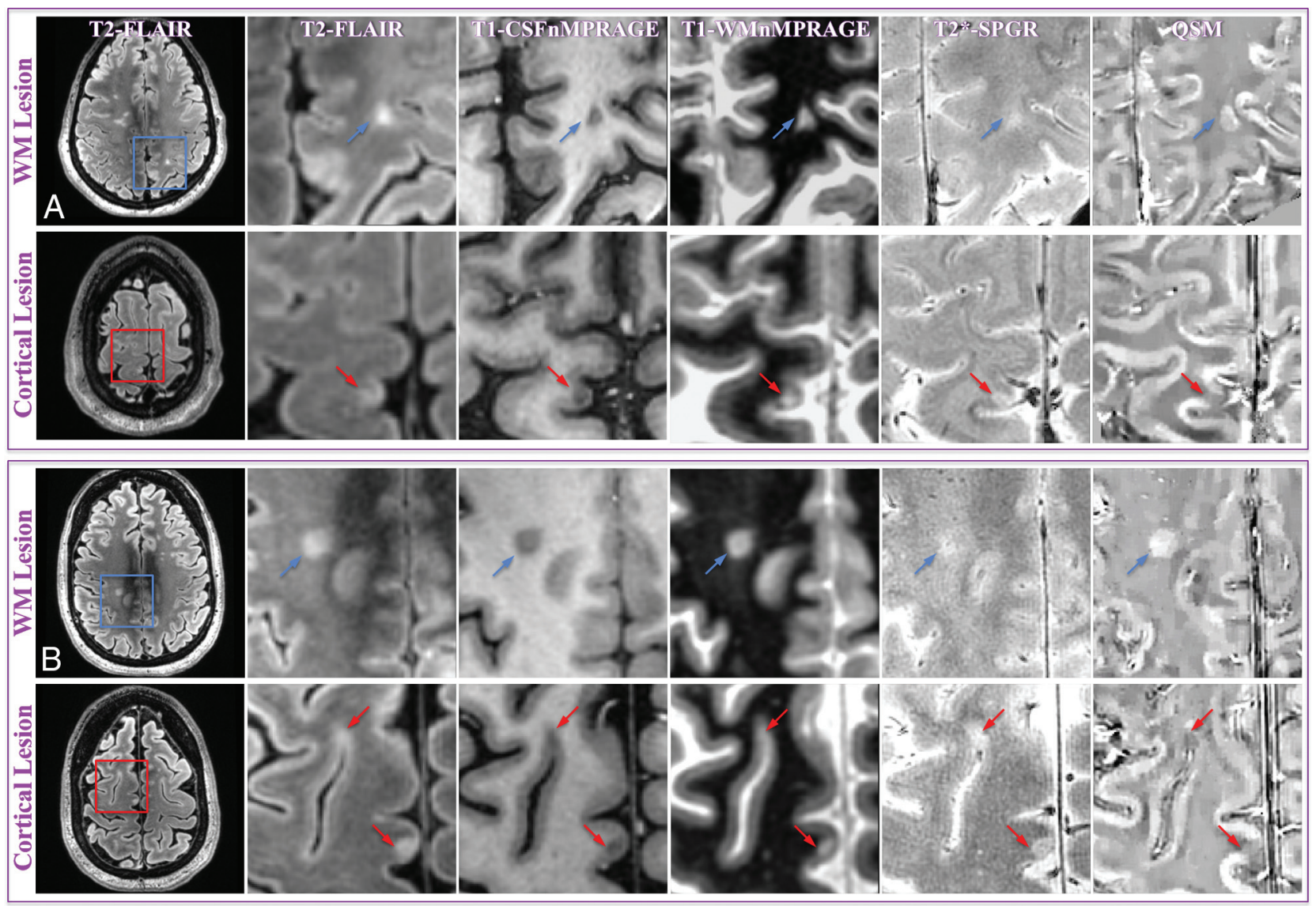

FIG 3. MR images of representative WM and cortical lesions from patients $4(A)$ and $5(B)$. A whole section of the T2-MPFLAIR image is displayed on the left column with a zoomed-in region (blue/red square) for all image contrasts. Two WM lesions (blue arrows) and 3 cortical lesions (red arrows) are shown. WM and cortical lesions are hyper- and hypointense relative to their adjacent parenchyma on QSM images, respectively, while both types of lesions show an identical contrast on all other images. CSFnMPRAGE indicates CSF-nulled MPRAGE; WMnMPRAGE, WM-nulled MPRAGE.

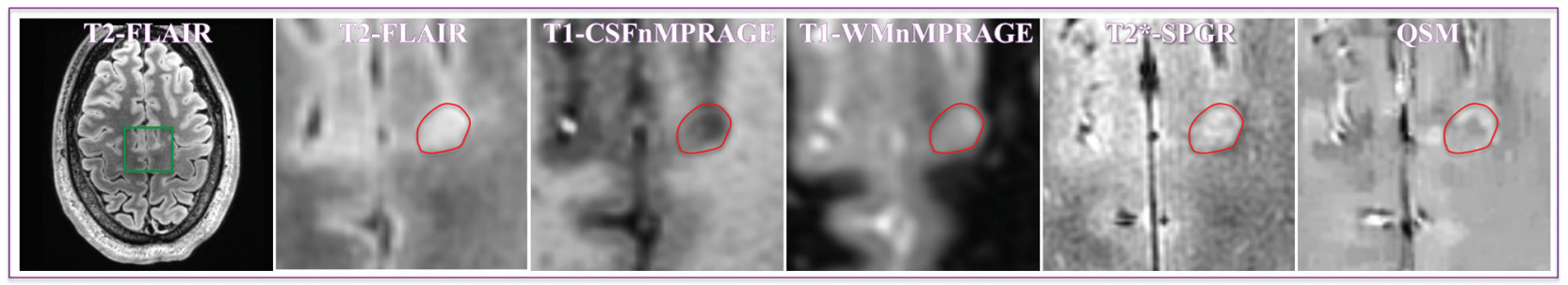

FIG 4. MR images of the only cortical lesion from patient 2. The lesion had a positive relative susceptibility and demonstrated a hyperintense core surrounded by an asymmetric hyperintense rim, suggesting that the lesion may have iron at its edge. Please see the Fig 3 legend for image descriptions.

Table 3: Mean lesion susceptibility relative to normal-appearing parenchyma ${ }^{a}$

\begin{tabular}{|c|c|c|c|c|}
\hline & \multirow[b]{2}{*}{ WM Lesions } & \multirow[b]{2}{*}{ Cortical Lesions } & \multicolumn{2}{|c|}{ Mixed Lesions } \\
\hline & & & WM Portion & Cortical Portion \\
\hline $\begin{array}{l}\text { Relative susceptibility } \\
\text { (ppm) (per lesion } \\
\text { type) }\end{array}$ & $0.014 \pm 0.014$ & $-0.018 \pm 0.013$ & $0.014 \pm 0.018$ & $-0.009 \pm 0.012$ \\
\hline$t$ test & $P<10^{-22}$ & $P<10^{-7}$ & $P<.043$ & $P<.054$ \\
\hline $\begin{array}{l}\text { Relative susceptibility } \\
\text { (ppm) (per lesion type } \\
\text { per subject) }\end{array}$ & $0.014 \pm 0.010$ & $-0.015 \pm 0.009$ & - & - \\
\hline$t$ test & $P<.0004$ & $P<.004$ & - & - \\
\hline
\end{tabular}

a The null hypothesis of the $t$ test is that the mean of relative susceptibility $=0$. The significance level is .0083 after correcting multiple comparisons of 6 using the Bonferroni method. the rim of acute and chronic active MS lesions, ${ }^{6,7,24,25}$ which can also contribute to an increased susceptibility. However, iron in most MS lesions will regress as disease duration increases, and in some inactive lesions, iron content could even be lower than that in normal-appearing WM. ${ }^{25}$ This may explain the presence of a few WM lesions with negative relative susceptibility. Nevertheless, because the susceptibility in most WM lesions was still positive relative to normal- 

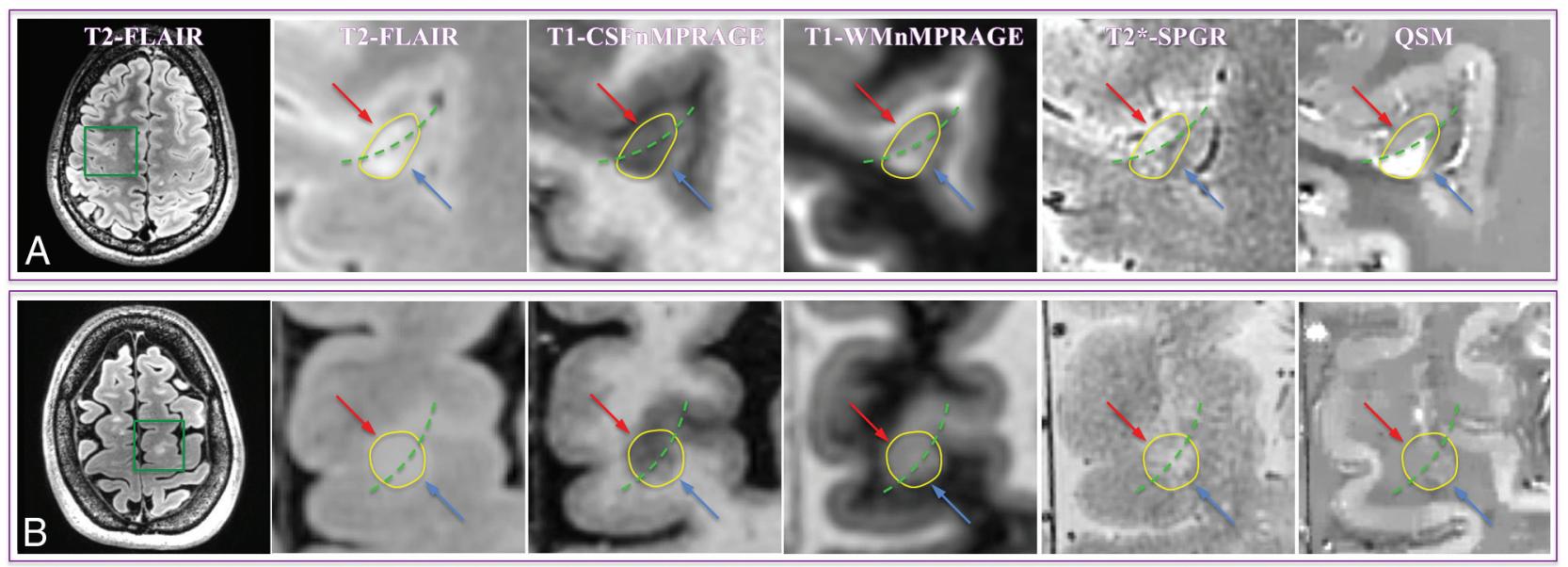

FIG 5. MR images of representative mixed lesions (yellow circles) from patients $1(A)$ and $6(B)$. The green dashed line divides a mixed lesion into its cortical (red arrow) and WM (blue arrow) components. A, The lesion has a QSM hypointense cortical portion and a hyperintense WM portion relative to adjacent normal-appearing GM and normal-appearing WM, respectively. $B$, The cortical component is hypointense compared with normal-appearing GM, while the white matter component is centrally isointense but peripherally slightly hyperintense compared with normalappearing WM. Please see the Fig 3 legend for image descriptions.

appearing WM in our current study and previous studies, ${ }^{9,23}$ it is likely that the effect of iron loss often does not completely offset that of demyelination.

\section{Negative Relative Susceptibility of Cortical Lesions}

In contrast to the positive relative susceptibility of WM lesions, the negative relative susceptibility of cortical lesions is counterintuitive, though supported by a recent postmortem study. ${ }^{22}$ Potential factors for susceptibility decrease are an iron decrease and/or a myelin increase. In theory, after initial demyelination, remyelination is possible in MS, but the regenerated myelin sheath is typically thinner than normal myelin ${ }^{26}$; this finding is consistent with overall reduced myelin relative to normal-appearing gray matter in histologic studies. ${ }^{10,27}$ Accompanying demyelination, loss of iron in cortical lesions has also been observed. ${ }^{10}$ The 2 contributions both lead to a decreased $\mathrm{R} 2^{*}$ (or increased T2*), as has been consistently reported in recent MR imaging studies. ${ }^{10-13}$ However, unlike their similar effects on $\mathrm{R} 2{ }^{*} / \mathrm{T} 2^{*}$, demyelination increases whereas the loss of iron decreases susceptibility. Therefore, in this particular case, QSM resolves a limitation of R2*/T2* mapping because it allows us to further conclude that in cortical lesions, iron loss dominates the susceptibility contrast over demyelination.

\section{Interpretation of the Different Susceptibility Contrast}

The different susceptibility contrast between cortical and WM lesions may be partly because the degree of myelination in the cortex is much less than that in WM, while the difference in their iron concentration is small. ${ }^{28}$ When both demyelination and iron loss are present, less iron loss is required to overwhelm demyelination in cortical lesions compared with WM. Indeed, in the cortex, iron has already been demonstrated to be the dominant source of MR susceptibility contrast and is well-correlated to both susceptibility and $\mathrm{R} 2{ }^{*} / \mathrm{T} 2{ }^{*} \cdot{ }^{29,30}$ Alternatively, this finding suggests that whenever there is increased iron in cortical lesions, a positive susceptibility value should be expected. The observation of iron accumulation in active cortical lesions has been reported in a previous study, ${ }^{27}$ and the positive susceptibility for cortical lesions did occur in our study. However, these positive susceptibility lesions were only $7.4 \%$ of all our cortical lesions. Although one may argue that this could be because most of our cortical lesions were in their chronic stage, in chronic WM lesions, an increased iron level can be maintained for several years. ${ }^{9,31}$ Moreover, recent results from $\mathrm{R} 2 * / \mathrm{T} 2^{\star}$ mapping consistently showed reduced $\mathrm{R} 2{ }^{\star}$ (or increased $\mathrm{T} 2{ }^{\star}$ ) in cortical lesions, ${ }^{10-13}$ and this could be even independent of disease stage. ${ }^{11,12}$ Thus, the underlying structural difference between the white matter and cortex alone may not explain all of the susceptibility difference, and pathologic changes that evolve differentially with time may also play a role.

Pathologically, an intact BBB and low degree of inflammation in cortical lesions suggest that there are fewer macrophages/microglia (either infiltrated or locally activated) than in WM lesions, especially at the active and chronic active phases. ${ }^{6,25}$ These cells phagocytize iron released from damaged oligodendrocytes and retain the iron in chronic WM lesions until the macrophages and microglia degenerate. ${ }^{25}$ Therefore, the paucity of these iron holders in cortical lesions may reduce the time interval for an increased iron level in these lesions. Thus, we speculate that compared with WM lesions, the time window for the initial phase of iron accumulation is narrower in cortical lesions due to their faster iron release secondary to the lack of inflammatory cells. This narrow time window could make it difficult for susceptibility-contrast MR imaging to depict the stage of iron accumulation in cortical lesions. Because free iron can cause oxidative neurodegeneration, ${ }^{25}$ the faster release of iron in cortical lesions may partly explain why cortical lesion load is more strongly correlated to the degree of neurodegeneration in MS. ${ }^{2}$ Nevertheless, this speculation warrants further investigation with longitudinal and contrast-enhanced studies.

Several limitations in our study should be addressed. First, due to the still low in vivo sensitivity of MR imaging to cortical lesions, ${ }^{10}$ our sampling of cortical lesions was likely incomplete and 
could be biased. The number of cortical lesions that can be analyzed can be further reduced after QSM reconstruction. Second, although we examined the lesion age by looking at the most recent clinically available contrast-enhanced 3T MR imaging, this can be imprecise for white matter and offers no information for cortical lesions. Given the lack of prior 7T imaging and/or concurrent gadolinium contrast administration, the acute nature of each individual lesion was not definitively ascertainable. Third, the relevance of the presumed iron loss in cortical lesions to clinical disability was not quantified. Last, QSM alone cannot distinguish the contribution of demyelination from that of an iron increase, which would often be seen at the acute phase of both cortical and WM lesions because QSM exhibits a positive sign in both cases, while $\mathrm{R} 2^{*}$ will decrease for demyelination and increase for iron deposition. To completely distinguish the susceptibility contributions from myelin and iron, our future studies will combine information from both QSM and R2* mapping.

\section{CONCLUSIONS}

QSM reveals an average negative magnetic susceptibility in cortical lesions and an average positive magnetic susceptibility in WM lesions, relative to their adjacent normal-appearing parenchyma. The negative susceptibility in cortical lesions suggests that iron loss dominates their susceptibility contrast. The different susceptibility contrast between cortical and WM lesions may reflect both their structural (degree of myelination) and pathologic (degree of inflammation) differences, in which the latter may lead to a faster release of iron in cortical lesions.

\section{ACKNOWLEDGMENTS}

The authors thank Dr Maged Goubran for his insightful discussion and suggestions in the preparation of the manuscript. We also extend our gratitude to the editor and 2 anonymous reviewers for their constructive comments, which greatly helped us improve the final version of the manuscript.

Disclosures: Thomas Tourdias—RELATED: Grant: Association pour la Recherche sur la Sclérose En Plaques, Labex Translational Research and Advanced Imaging Laboratory. May Han—UNRELATED: Consultancy: Pfizer. Yi Wang—RELATED: Grant: National Institutes of Health (R01NS090464)*; UNRELATED: Patents (planned, pending or issued): Cornell University, ${ }^{*}$ Comments: one of the inventors on the QSM patent; OTHER RELATIONSHIPS: in discussion with Cornell regarding QSM technologybased startup. Brian Rutt—RELATED: Grant: GE Healthcare.* Michael M. ZeinehUNRELATED: Grants/Grants Pending: Doris Duke Charitable Foundation,* Dana Foundation, ${ }^{*}$ Radiological Society of North America, ${ }^{*}$ Other: GE Healthcare, Comments: some research support.* *Money paid to the institution.

\section{REFERENCES}

1. Calabrese M, Filippi M, Gallo P. Cortical lesions in multiple sclerosis. Nat Rev Neurol 2010;6:438-44 CrossRef Medline

2. Harrison DM, Roy S, Oh J, et al. Association of cortical lesion burden on 7-T magnetic resonance imaging with cognition and disability in multiple sclerosis. JAMA Neurol 2015;72:1004-12 CrossRef Medline

3. Peterson JW, Bo L, Mörk S, et al. Transected neurites, apoptotic neurons, and reduced inflammation in cortical multiple sclerosis lesions. Ann Neurol 2001;50:389-400 CrossRef Medline

4. van Horssen J, Brink BP, de Vries HE, et al. The blood-brain barrier in cortical multiple sclerosis lesions. J Neuropathol Exp Neurol 2007; 66:321-28 CrossRef Medline
5. Louapre C, Lubetzki C. Neurodegeneration in multiple sclerosis is a process separate from inflammation: yes. Mult Scler 2015;21: 1626-28 CrossRef Medline

6. Bagnato F, Hametner S, Yao B, et al. Tracking iron in multiple sclerosis: a combined imaging and histopathological study at 7 Tesla. Brain 2011;134:3602-15 CrossRef Medline

7. Yao B, Bagnato F, Matsuura E, et al. Chronic multiple sclerosis lesions: characterization with high-field-strength MR imaging. $\mathrm{Ra}$ diology 2012;262:206-15 CrossRef Medline

8. Wisnieff C, Ramanan S, Olesik J, et al. Quantitative susceptibility mapping (QSM) of white matter multiple sclerosis lesions: interpreting positive susceptibility and the presence of iron. Magn Reson Med 2015;74:564-70 CrossRef Medline

9. Chen W, Gauthier SA, Gupta A, et al. Quantitative susceptibility mapping of multiple sclerosis lesions at various ages. Radiology 2014;271:183-92 CrossRef Medline

10. Yao B, Hametner S, van Gelderen $P$, et al. 7 Tesla magnetic resonance imaging to detect cortical pathology in multiple sclerosis. PLoS One 2014;9:e108863 CrossRef Medline

11. Mainero C, Louapre C, Govindarajan ST, et al. A gradient in cortical pathology in multiple sclerosis by in vivo quantitative $7 \mathrm{~T}$ imaging. Brain 2015;138:932-45 CrossRef Medline

12. Louapre C, Govindarajan ST, Gianni C, et al. Beyond focal cortical lesions in MS: an in vivo quantitative and spatial imaging study at 7T. Neurology 2015;85:1702-09 CrossRef Medline

13. Jonkman LE, Fleysher L, Steenwijk MD, et al. Ultra-high field MTR and $\mathrm{qR} 2 *$ differentiates subpial cortical lesions from normal-appearing gray matter in multiple sclerosis. Mult Scler 2015 Dec 16. [Epub ahead of print] Medline

14. Deh K, Nguyen TD, Eskreis-Winkler S, et al. Reproducibility of quantitative susceptibility mapping in the brain at two field strengths from two vendors. J Magn Reson Imaging 2015;42:1592600 CrossRef Medline

15. Wang Y, Liu T. Quantitative susceptibility mapping (QSM): decoding MRI data for a tissue magnetic biomarker. Magn Reson Med 2015;73:82-101 CrossRef Medline

16. Liu C, Li W, Tong KA, et al. Susceptibility-weighted imaging and quantitative susceptibility mapping in the brain. J Magn Reson Imaging 2015;42:23-41 CrossRef Medline

17. McDonald WI, Compston A, Edan G, et al. Recommended diagnostic criteria for multiple sclerosis: guidelines from the International Panel on the Diagnosis of Multiple Sclerosis. Ann Neurol 2001;50: 121-27 CrossRef Medline

18. Saranathan M, Tourdias T, Bayram E, et al. Optimization of whitematter-nulled magnetization prepared rapid gradient echo (MPRAGE) imaging. Magn Reson Med 2015;73:1786-94 CrossRef Medline

19. Saranathan M, Tourdias T, Kerr AB, et al. Optimization of magnetization-prepared 3-dimensional fluid attenuated inversion recovery imaging for lesion detection at 7 T. Invest Radiol 2014;49:290-98 CrossRef Medline

20. Liu J, Liu T, de Rochefort L, et al. Morphology enabled dipole inversion for quantitative susceptibility mapping using structural consistency between the magnitude image and the susceptibility map. Neuroimage 2012;59:2560-68 CrossRef Medline

21. Smith SM, Jenkinson M, Woolrich MW, et al. Advances in functional and structural MR image analysis and implementation as FSL. Neuroimage 2004;23(suppl 1):S208-19 CrossRef Medline

22. Wisnieff C, Ryan R, Pitt D, et al. Investigation of susceptibility contrast in grey and white matter multiple sclerosis lesions. In: Proceedings of the Annual Scientific Meeting of the International Society for Magnetic Resonance in Medicine, Milan, Italy. May 1016,2014

23. Li X, Harrison DM, Liu H, et al. Magnetic susceptibility contrast variations in multiple sclerosis lesions. J Magn Reson Imaging 2016; 43:463-73 CrossRef Medline

24. Mehta V, Pei W, Yang G, et al. Iron is a sensitive biomarker for 
inflammation in multiple sclerosis lesions. PLoS One 2013;8:e57573 CrossRef Medline

25. Hametner S, Wimmer I, Haider L, et al. Iron and neurodegeneration in the multiple sclerosis brain. Ann Neurol 2013;74:848-61 CrossRef Medline

26. Chari DM. Remyelination in multiple sclerosis. Int Rev Neurobiol 2007;79:589-620 CrossRef Medline

27. Pitt D, Boster A, Pei W, et al. Imaging cortical lesions in multiple sclerosis with ultra-high-field magnetic resonance imaging. Arch Neurol 2010;67:812-18 CrossRef Medline

28. Haacke EM, Cheng NY, House MJ, et al. Imaging iron stores in the brain using magnetic resonance imaging. Magn Reson Imaging 2005; 23:1-25 CrossRef Medline

29. Fukunaga M, Li TQ, van Gelderen P, et al. Layer-specific variation of iron content in cerebral cortex as a source of MRI contrast. Proc Natl Acad Sci U S A 2010;107:3834-39 CrossRef Medline

30. Langkammer C, Krebs N, Goessler W, et al. Susceptibility induced gray-white matter MRI contrast in the human brain. Neuroimage 2012;59:1413-19 CrossRef Medline

31. Bian $\mathrm{W}$, Harter $\mathrm{K}$, Hammond-Rosenbluth $\mathrm{KE}$, et al. A serial in vivo 7T magnetic resonance phase imaging study of white matter lesions in multiple sclerosis. Mult Scler 2013;19:69-75 CrossRef Medline 